\title{
Pump-probe localization technique of varying solid contacts
}

M. Terzi, L. Chehami, M. Farin, E. Moulin, V. Aleshin, N. Smagin, J. de Rosny, and F. Benmeddour

Citation: The Journal of the Acoustical Society of America 149, 2943 (2021); doi: 10.1121/10.0004820

View online: https://doi.org/10.1121/10.0004820

View Table of Contents: https://asa.scitation.org/toc/jas/149/5

Published by the Acoustical Society of America

\section{ARTICLES YOU MAY BE INTERESTED IN}

Seminal article about model-based space-time array processing

The Journal of the Acoustical Society of America 149, R9 (2021); https://doi.org/10.1121/10.0004816

Visual geometry Group-UNet: Deep learning ultrasonic image reconstruction for curved parts

The Journal of the Acoustical Society of America 149, 2997 (2021); https://doi.org/10.1121/10.0004827

Homogenization of periodic 1-3 piezocomposite using wave propagation: Toward an experimental method The Journal of the Acoustical Society of America 149, 3122 (2021); https://doi.org/10.1121/10.0004824

Simulation of pulsed ultrasonic diffraction in viscous fluids using transmission line matrix method

The Journal of the Acoustical Society of America 149, 2988 (2021); https://doi.org/10.1121/10.0004825

Nearly 40 years of domination by electret microphones

The Journal of the Acoustical Society of America 149, R7 (2021); https://doi.org/10.1121/10.0003934

Modelling and measurement of laser-generated focused ultrasound: Can interventional transducers achieve therapeutic effects?

The Journal of the Acoustical Society of America 149, 2732 (2021); https://doi.org/10.1121/10.0004302 


\title{
Pump-probe localization technique of varying solid contacts
}

\author{
M. Terzi, ${ }^{1}$ L. Chehami, ${ }^{1}$ M. Farin, ${ }^{2}$ E. Moulin, ${ }^{1, a), b)}$ V. Aleshin, ${ }^{1, c)}$ N. Smagin, ${ }^{1}$ J. de Rosny, ${ }^{2}$ and F. Benmeddour ${ }^{1, a)}$ \\ ${ }^{1}$ Université de Lille, Centre National de la Recherche Scientifique, Centrale Lille, Université Polytechnique Hauts-de-France, \\ Unité Mixte de Recherche 8520, Institut d'Électronique de Microélectronique et de Nanotechnologie, F-59000 Lille, France \\ ${ }^{2}$ Institut Langevin, Centre National de la Recherche Scientifique, Unité Mixte de Recherche 7587, F-75231 Paris, France
}

\begin{abstract}
:
A baseline-free defect localization method in thin plates is proposed and tested. In this proof-of-concept work, a steel ball pressed against an aluminum plate is used to mimic a surface contact defect. The technique takes benefit of a repetitive nonlinear pump-probe interaction with a backpropagation imaging algorithm. High-frequency probe waves are periodically emitted by a piezoelectric patch transducer glued to the plate. Propagated flexural waves are recorded using a distributed array of transducers. At the same time, a continuous low-frequency pump vibration provided by a shaker fixed to the plate modulates the contact state. By combining multiple probe signals, the contact can be successfully localized. Contrast of the localization images is finally improved by a factor of 3 to 5 by implementing a modified version based on synchronous detection of the imaging algorithm.
\end{abstract}

(C) 2021 Acoustical Society of America. https://doi.org/10.1121/10.0004820

(Received 14 October 2020; revised 9 April 2021; accepted 11 April 2021; published online 3 May 2021)

[Editor: Marcel C. Remillieux]

Pages: 2943-2949

\section{INTRODUCTION}

Structural health monitoring (SHM) refers to a permanent inspection of a system or structure in service and includes damage detection, and structural integrity assessment, as well as damage evolution prognostics and lifetime estimations. Permanent monitoring should be capable of detecting fatigue damage that appears in materials under repetitive loading and possibly locate it. Many SHM techniques require additional reference measurements on a baseline intact sample for comparison with the actual monitored sample. However, even a significant difference from baseline in measured properties does not necessarily indicate the presence of damage. Indeed, materials' characteristics can slowly evolve due to aging, environmental effects, etc. In addition, measurements in the original state of a structure are frequently not available or not possible.

In this work, the interest is focused on the case of plateshaped structures, for which a few baseline-free (or reference-free) methods have been proposed recently in the literature. ${ }^{1-7}$

One of the attempts to reduce baseline data amounts consists of using a so-called instantaneous baseline. ${ }^{1}$ In this approach, Lamb wave propagation along several paths is interrogated in pitch-catch configuration with a distributed transducers array. Common features in the undamaged paths are considered as instantaneous baseline and used for damage prediction.

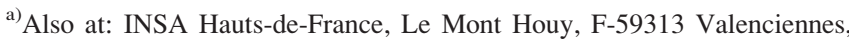
France.

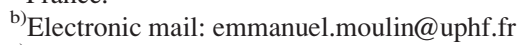

${ }^{c}$ Also at: Tomsk State University, 634050, Tomsk, Russia.
}

Time reversibility of Lamb waves ${ }^{2,3}$ allows one to use the time reversal (TR) method for damage detection. Damage index is used to evaluate the difference between input and TR reconstructed signals for the distributed sensor array. The threshold for damage index distinguishing undamaged and damaged paths was established comparing damage indices of the paths with each other, not with a baseline. Dividing into forward and backward propagating waves and applying a standing wave filter allows damage to be visualized. It was concluded ${ }^{5}$ that TR is relatively immune to environmental and operational variations but suffers from significant limitations such as necessity to employ narrowband signals to minimize the dispersion and a large number of transducers required for precise localization. A similar approach relies on violating the reciprocity principle in the context of delamination detection. ${ }^{6}$

Another idea is applied in an instantaneous baseline group of methods based on mode conversion. ${ }^{7}$ It benefits from polarization characteristics of collocating PZT transducers attached to the opposite surfaces of a thin plate. Guided waves excited across different transducer pairs allow damage identification.

Evidences of interaction between independent waves of different frequencies is a classical manifestation of nonlinearity existence. For example, pump-probe experiments applied together with coda wave interferometry results in coda wave velocity variations due to the interaction with low-frequency pump waves. ${ }^{8,9}$ Coda wave interferometry is a highly sensitive damage detection method, however, it does not provide localization information.

In this paper, a baseline-free imaging method capable of localizing contact acoustic nonlinearity (CAN) in thin plates is described. This method is based on a pump-probe 
interaction, in which information of damage is provided by differences in high frequency (HF) probe signals measured under varying contact conditions induced by low frequency (LF) pumping. The contact defect is localized by using a backpropagation technique presented in previous works, ${ }^{10-12}$ where baseline-subtraction allowed strong enhancement of the scattering signature of the defect.

In the reported experiments, a steel ball pressed against an aluminum plate is used to mimic a CAN. Similar to a crack or delamination, the ball excited by the LF pumping slightly modifies the propagation conditions for the HF probe wave. These weak changes are considerably enhanced by cumulating effects over multiple different contact states.

To be precise, the nonlinearity in this study resides in the interaction of the pump vibrations with the solid contacts in the "defect." Indeed, the modulation of the defect properties induced by the pump vibration can be seen as a local manifestation of acousto-elastic or nonlinear-elasticity effects. ${ }^{13-16}$ But the detection process does not assume any nonlinear interaction between the ultrasound (probe) wave and the defect.

Sections II and III describe in details the experimental procedure and signal processing for defect localization, respectively. Finally, results of localization images are presented and discussed in Sec. IV, before concluding.

\section{EXPERIMENTAL SETUP AND PUMP-PROBE PRINCIPLE}

In the presented experiment, the setup consists of a thin aluminum rectangular plate $(1 \mathrm{~m} \times 0.5 \mathrm{~m} \times 3 \mathrm{~mm})$ horizontally suspended with elastic strings on a metallic supporting structure and instrumented with bonded piezoelectric PZT transducers (model "KEPO FT-20T-4.0A1-465") distributed over its surface. Instead of a real defect we use a $1 \mathrm{~cm}$ diameter steel ball pressed against the top plate surface with a metallic blade fixed on the same support (see Fig. 1).

Two types of vibrations are generated in the plate (Fig. 2): a high-frequency (HF) probe wave corresponding to a one-period sine toneburst at $f_{\text {probe }}=10 \mathrm{kHz}$, sequentially emitted at regular time-intervals $\Delta T$; and a low-frequency (LF) harmonic pump vibration $s_{\text {pump }}(t)$ with a frequency $f_{\text {pump }}$ in the range of a few $\mathrm{Hz}\left(f_{\text {pump }} \ll f_{\text {probe }}\right)$. The probe signal is emitted using one of the piezoelectric patch transducers glued to the plate surface. The pump vibration is applied to the plate by means of a shaker (TIRAvib 50018, fed through its power amplifier TIRA BAA60) connected to the plate with a steel rod equipped at its extremity with a polystyrene foam-coated clamp gripped to the plate edge. In that way, the shaker forces alternative normal motion at the clamp point of the plate.

In addition to the emitting piezoelectric transducer, 14 receiving patch transducers of the same kind are bonded at known positions. Signals from these transducers are recorded by means of a multi-channel digital sampling board (24 I/O MOTU, $96 \mathrm{kS} / \mathrm{s}, 24$ bits) for $40 \mathrm{~s}$. Low-pass and high-pass filtering easily allow the retrieval of the LF pump vibration and the HF probe signals, respectively, from

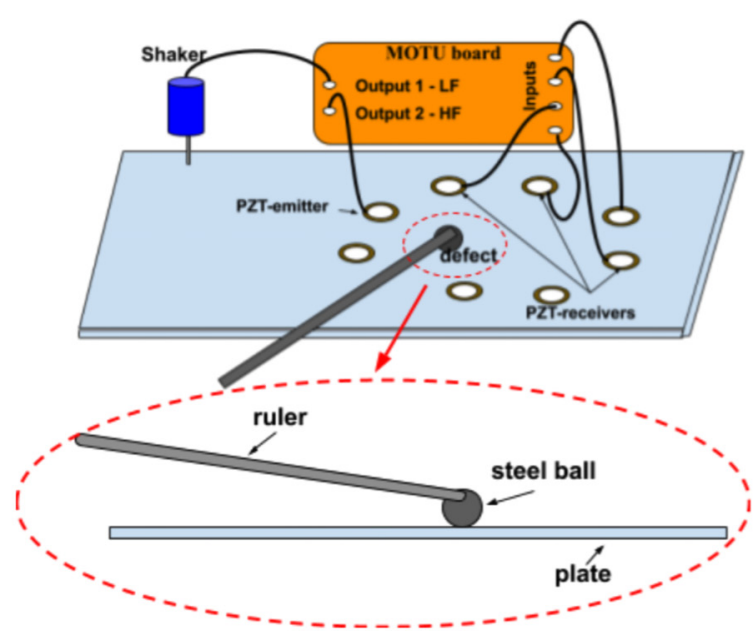

(a)

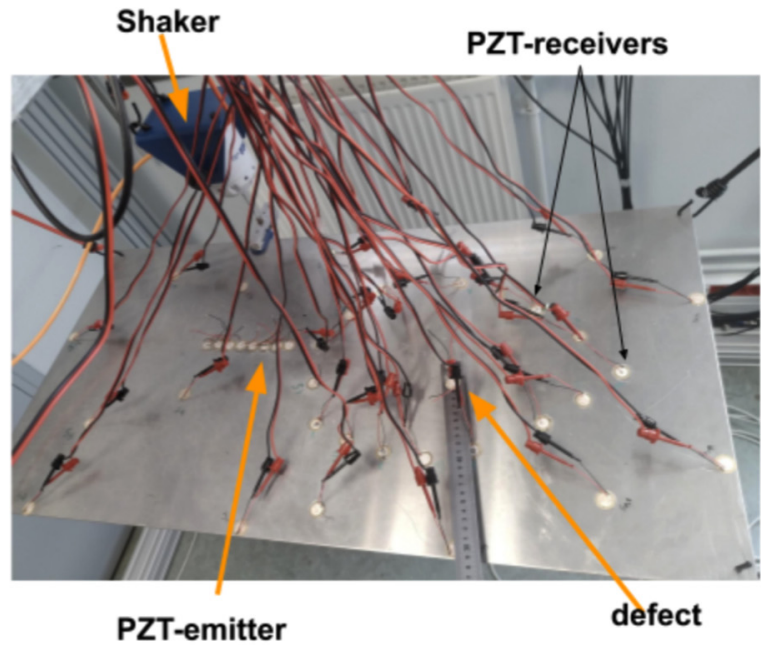

(b)

FIG. 1. (Color online) Experimental setup for pump-probe experiment. (a) Schematic drawing. (b) Picture of the experimental bench.

these recorded signals. Individual HF signals are then obtained by cutting high-pass filtered signals from each sensor into slices of duration $\Delta T$. The HF signal in the $m$ th slice from the $n$th receiver $\mathrm{R}_{n}$ is thus noted $s_{m, n}(t)$. Note that the relative amplitudes associated with pump and probe waves

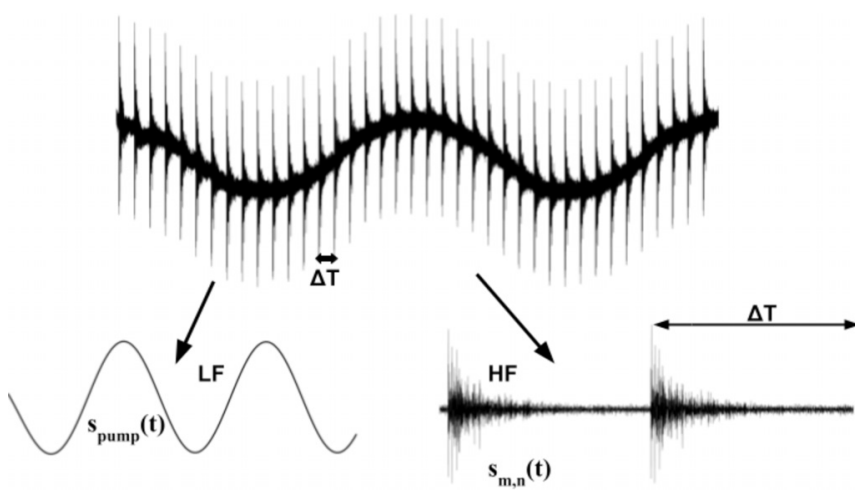

FIG. 2. Representation of recorded signals as a mix of the LF pump and HF probe waves, and associated notations. 
in the waveform displayed in Fig. 2 do not reflect their actual strengths, since the transducers' bandpass is adapted to the probe frequencies and misadapted to the pump. Indeed, as will be seen below, strains induced by the pump vibrations are around three order of magnitudes higher than strains induced by the HF probe wave.

The idea behind this experimental setup is that each recorded HF signal, containing components with wave paths having crossed the contact area, will capture information on the contact state at the instant of measurement. Indeed, $\Delta T$ being much smaller than the LF period, as pointed out above, the contact state can be assumed to be stationary during one HF signal slice. Since LF pump vibrations will continuously and cyclically modulate the contact condition (contact force and relative positions) between the plate and the ball, the HF acquisition will sample these different contact conditions with a rate corresponding to $1 /\left(\Delta T \times f_{\text {pump }}\right)$ samples per period.

Since the effect of contact modulation in the HF signals is expected to be slight, we seek to cumulate information acquired at different $\mathrm{HF}$ samples (i.e., different HF signal slices and therefore different contact states). Details are given in Sec. III.

\section{SIGNAL PROCESSING FOR LOCALIZATION OF CONTACT MODULATIONS}

Differential signals $\Delta s_{m, n}$ are defined by subtracting the extracted HF signals $s_{m, n}$ and their averages over all $M$ slices,

$$
\Delta s_{m, n}(t)=s_{m, n}(t)-\frac{1}{M} \sum_{i=1}^{M} s_{i, n}(t) .
$$

The average term plays here the role of a reference state, corresponding to the average defect contribution (static platedefect contact case). The differential signals $\Delta s_{m, n}$ thus essentially contain defect-related signatures due to the modulations around this average defect contribution induced by the pump.

The defect localization procedure consists in constructing an image by processing these differential signals pixel by pixel, using a backpropagation-based procedure already exploited with success in previous works. ${ }^{10}$ For clarity, the main steps of this processing are briefly described below.

First, for a given pixel of coordinates $(x, y)$ and a given signal slice $m$, differential signals are backpropagated over distances $d_{n}(x, y)$ between the emitter and the $n$th receiver via the pixel. This is simply done through multiplication in the frequency domain by the inverse propagation phase term

$$
F_{m, n}(x, y, \omega)=\Delta s_{m, n}(\omega) e^{j k(\omega) d_{n}(x, y)},
$$

where the wavenumber $k$ fulfills the dispersion relation of $\mathrm{A}_{0}$ Lamb mode (flexural wave). Indeed, since the frequency-thickness range is below the cut-off frequency of higher-order Lamb modes, the only possible mode other than the flexural antisymmetric $A_{0}$ one is the symmetric $S_{0}$ mode. But here, surfacic and asymmetric emission and reception by the transducers are not adapted to the essentially compressional symmetric mode. This can be easily verified by observing the signal waveforms, on which the first (in terms of time-of-arrival) significant wavepackets match the $\mathrm{A}_{0}$ group velocity. This has been validated in previous works in similar excitation and reception conditions, ${ }^{10,11}$ showing that ignoring $\mathrm{S}_{0}$ component in the backpropagation process is perfectly acceptable.

Then we construct so-called backpropagation function by summation over all receivers, ${ }^{10}$

$$
b p f_{m}(x, y, \omega)=\sum_{n=1}^{N_{r}} F_{m, n}(x, y, \omega),
$$

where $N_{r}$ states for a number of receivers. For pixels coinciding with the defect position, the backpropagation process will perfectly compensate for propagation phases and, back in the time-domain, the backpropagation function $b p f_{m}(x, y, t)$ will result in a constructive sum at zero time. On the contrary, for pixels at arbitrary positions, summed terms will have no particular phase relation. Therefore, the pixel intensity defined as

$$
I_{m}(x, y)=\int_{-T_{0} / 2}^{T_{0} / 2}\left[b p f_{m}(x, y, t)\right]^{2} \mathrm{~d} t
$$

is expected to exhibit a local maximum at the defect location. Here, $T_{0}$ is the duration of the emitted wavepacket (typically the inverse of the bandwidth), and integration over the interval $\left[-T_{0} / 2, T_{0} / 2\right]$ allows one to eliminate the influence of reverberations outside this interval.

Finally, to enhance detection quality and reduce noise effects, averaging over all $M$ slices is performed, resulting in

$$
I_{\mathrm{inc}}(x, y)=\frac{1}{M} \sum_{m=1}^{M} I_{m}(x, y)
$$

where the subscript "inc" stands for "incoherent," since the final image is made up of incoherent summation (sum of energy terms) over all HF signal slices.

In accordance with the formulated principle, $I_{\text {inc }}(x, y)$ reaches a maximum when $(x, y)$ coincides with the defect position. A graphical representation of $I_{\text {inc }}(x, y)$ thus constitutes a localization image of the defect, as will be confirmed in Sec. IV.

In a refined version of the procedure, the backpropagation functions obtained for each HF slice can be coherently summed. To that end, we define

$$
B P F(x, y, t)=\frac{1}{M} \sum_{m=1}^{M} a_{m} b p f_{m}(x, y, t),
$$

where $a_{m}$ is a set of ponderation coefficients aiming at ensuring that the summation will be constructive. 
Indeed, since the contact at the defect location is continuously and alternatively modulated through the LF shaker excitation $s_{\text {pump }}(t)$, averaging with unitary ponderation $(a m=1)$ should cancel the defect contribution. Instead, we will apply here the well-known principle of synchronous detection, by defining ponderation as

$$
a_{m}=s_{\text {pump }}\left(T_{m}\right)
$$

where $T_{m}=m \Delta T$ is the instant of the $m$ th HF emission (slice $m$ ). As explained above, the pump vibration $s_{\text {pump }}$ is easily extracted by low-pass filtering the received signals.

Then, similar to Eq. (4), an image can be constructed from coherently summed backpropagation functions as

$$
I_{\mathrm{coh}}(x, y)=\int_{-T_{0} / 2}^{T_{0} / 2}[B P F(x, y, t)]^{2} \mathrm{~d} t .
$$

\section{RESULTS AND DISCUSSION}

First, the results of the measurements and signal processing resulting in Eq. (5) (incoherent imaging) are shown in Fig. 3. Time interval between HF signal emissions is set as $\Delta T=50 \mathrm{~ms}$. The pump signal is a sine waveform of

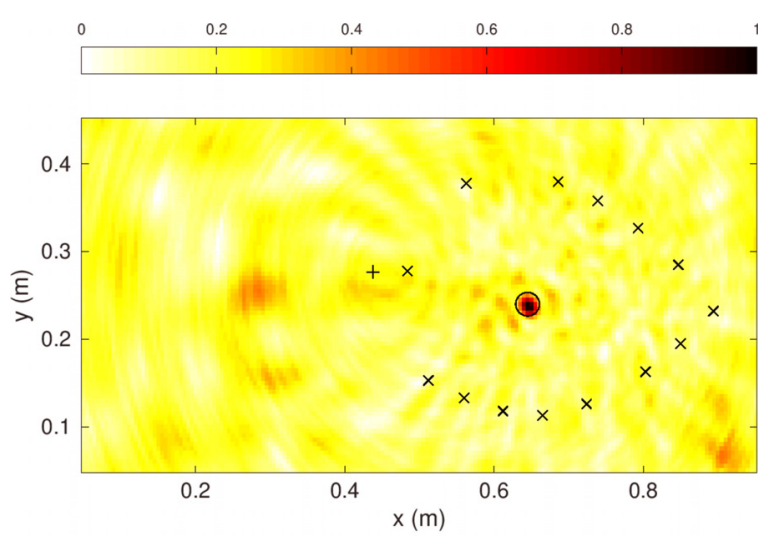

(a)

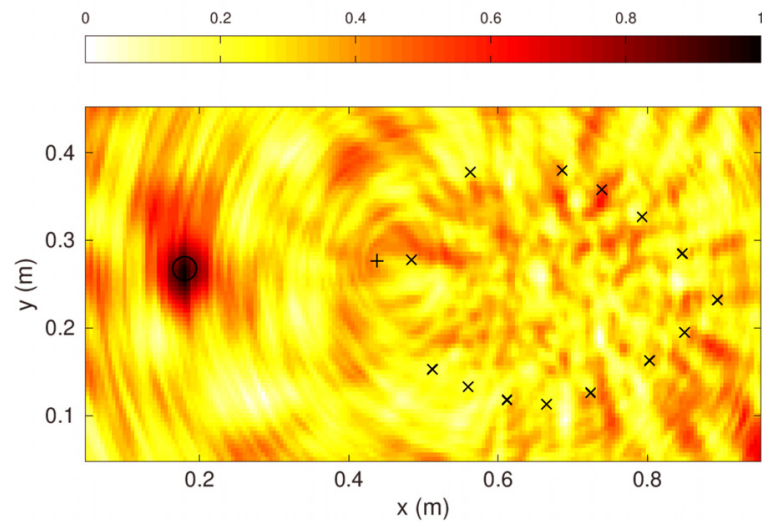

(b)

FIG. 3. (Color online) Defect localization images resulting from incoherent process $I_{\text {inc }}(x, y)$ in Eq. (5). True defect positions are indicated by the small black circles. HF probe emitter is indicated by + and $\mathrm{HF}$ receivers $\mathrm{R}_{n}$ are indicated by $\times$. (a) Defect coordinates $(0.645,0.24)$. (b) Defect coordinates $(0.18,0.268)$. amplitude $3 \mathrm{~V}$ and frequency $f_{\text {pump }}=1 \mathrm{~Hz}$, fed to the shaker's power amplifier. The emitted HF signal is a one-period sine tone burst at $f_{\text {probe }}=10 \mathrm{kHz}$ and $10 \mathrm{~V}$ amplitude. Camera recordings of the steel ball motion and normal velocity measurement using a laser vibrometer allowed us to quantify the pump- and probe-induced strains corresponding to these experimental conditions (see Appendixes A and B for technical details). Estimated values of the strain amplitudes at the defect location are on the order of $1.75 \times 10^{-5}$ for the pump vibrations and $1.3 \times 10^{-8}$ for the probe wave.

In the figures, the defect position is marked with a black circle, the black crosses correspond to receivers $\mathrm{R}_{n}$ and the black + symbol to the HF emitter. Two defect positions are tested: inside [Fig. 3(a)] and outside [Fig. 3(b)] the receiver array.

Both defect positions are correctly detected through localized higher-intensity spots on the images. The fact that the spot corresponding to the defect outside the receiver array is larger than the one inside is coherent with the wellknown diffraction laws relative to the array aperture.

There is an apparent speckle-like background noise in both images. It is mostly related to incoherent averaging of intensities through Eqs. (4) and (5), which does not suppress all of the experimental noise and reverberation contributions.

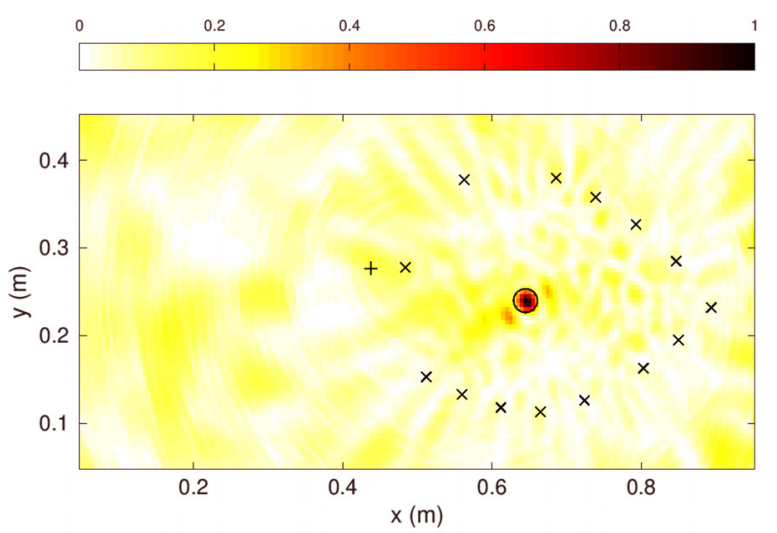

(a)

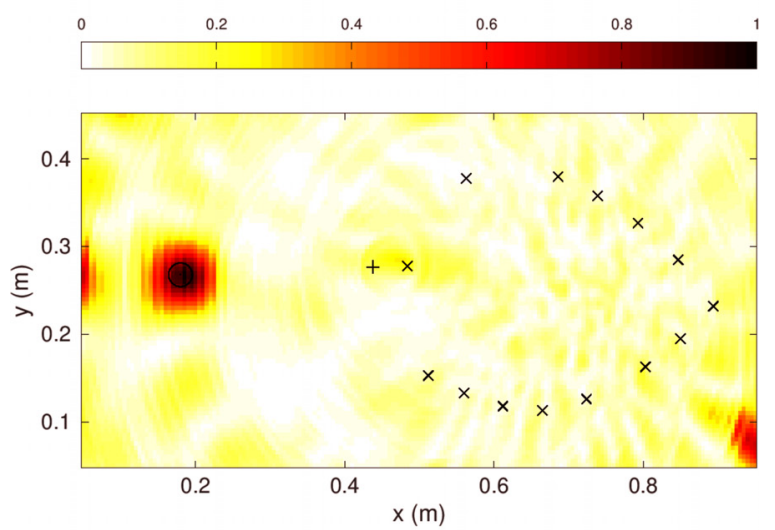

(b)

FIG. 4. (Color online) Defect localization images resulting from coherent process $I_{\text {coh }}(x, y)$ in Eq. (8). True defect positions are indicated by the small black circles. HF probe emitter is indicated by + and $\mathrm{HF}$ receivers $\mathrm{R}_{n}$ are indicated by $\times$. (a) Defect coordinates $(0.645,0.24)$. (b) Defect coordinates $(0.18,0.268)$. 
To remedy that, coherent images [Eq. (8)] obtained from the same experimental recordings have been computed as well. They are shown in Fig. 4. The benefit of synchronous detection application, resulting in coherent and constructive summation of the backpropagation function, on the image quality is obvious.

For more quantitative comparison of coherent and incoherent images, image contrast is defined as

$$
C=\frac{I\left(x_{d}, y_{d}\right)-I_{\mathrm{av}}}{I_{\mathrm{av}}},
$$

where $\left(x_{d}, y_{d}\right)$ are the defect coordinates and $I_{\mathrm{av}}$ is the average intensity value of the image. $I$ is either $I_{\text {inc }}$ or $I_{\text {coh }}$ from Eqs. (5) and (8). This is a classical definition corresponding to so-called Weber contrast.

Computed contrasts for incoherent and coherent images for the first defect location [Figs. 3(a) and 4(a)] are $C_{\text {inc }}$ $=4.7$ and $C_{\text {coh }}=14.8$, respectively. For the second defect location [Figs. 3(b) and 4(b)] they are $C_{\text {inc }}=2.4$ and $C_{\text {coh }}=12.2$. This corresponds to a factor 3 to 5 of contrast improvement when applying the pump-probe technique in a synchronous coherent way.

Finally, by performing additional recordings with different shaker input amplitudes, as explained in Appendix A, we have observed that the defect is detected on the images only for pump-strain values higher than a threshold on the order of $10^{-5}$. This point will be more deeply investigated in future works.

\section{CONCLUSION}

A baseline-free defect detection method in thin plates is proposed, based on a repetitive pump-probe experimental procedure for modulating the contact interface inside a defect. These modulations are detected through interaction with ultrasound waves propagated and received on a distributed transducer array. A backpropagation algorithm applied to differential ultrasound signals (subtraction of average modulation state) then provides localization images of the defect. In the preliminary experiment presented here, a steel ball pressed against the plate surface is used instead of an actual defect such as cracks or delaminations. Two variants of the signal processing procedure are tested: incoherent and coherent imaging, corresponding to asynchronous (sum of energies) and synchronous detection (summation after ponderation by the pump amplitudes), respectively. For pump modulations corresponding to strain variations higher than $10^{-5}$, both variants have allowed unambiguous localization of defects. However, without surprise, the coherent version results in significantly higher image contrasts.

In future work, we intend to test the method for actual cracks with internal contacts. More precise quantification of parameters influencing the detection and contrast of localization images will also be required. In addition to strain characterization at the contact location, another insightful parameter to be quantified is the instantaneous value of the scattering cross section at the instant of the probe measurement, which will be a direct indicator of the probepump interaction. We have recently developed an original method based on coda analysis that should allow such characterization. ${ }^{17}$ In a similar vein, a study of the influence of the evolution of contact properties in the defect (in particular, the more or less nonlinear behavior of the pump-defect interaction) would be worthwhile. Controlling the offset contact force could be a convenient way to do it, or alternatively using other types of defects such as a bolt tightened at different torques. ${ }^{18}$

Finally, a further idea would be to adapt the developed procedure for passive SHM, using the ambient vibrations of a structure accompanying its operation as the low-frequency pump. Indeed, the fact that even incoherent processing provides exploitable results (as shown in Fig. 3) is promising for such applications where uncontrolled vibrations could be used as the pump.

\section{ACKNOWLEDGMENTS}

This research was supported by the French National Research Agency ANR, Grant No. ANR-17-CE08-0013-01 (PANSCAN project). One of the authors (V.A.) particularly acknowledges the support from the Tomsk State University competitiveness improvement program.

\section{APPENDIX A: ESTIMATE OF THE PUMP-INDUCED STRAIN IN THE DEFECT}

As explained, the defect is a contact area between two elastic solids: a steel ball and the plate [Fig. 1(a)]. The contact force $F$ between them is prescribed through the deflection of an elastic steel ruler. Therefore, $F$ can be related to the ball vertical displacement $d$ from rest position (without contact) through the well-known cantilever beam relations ${ }^{19}$

$$
F=\frac{E_{s} b h^{3} d}{4 L^{3}}
$$

with $E_{s}$ the Young's modulus of the ruler material (steel here) and $h, b$, and $L$ the ruler's thickness, width, and length, respectively.

Hertz's theory of contact then provides the maximum pressure applied by the steel ball to the plate surface as ${ }^{20}$

$$
p_{0}=\frac{1}{\pi}\left(\frac{6 F E^{* 2}}{R^{2}}\right)^{1 / 3}
$$

where $R$ is the ball radius and $E^{*}=E E_{s} /\left[\left(1-\nu_{s}^{2}\right) E\right.$ $+\left(1-\nu^{2}\right) E_{s}$ ], with $E, \nu$ the Young's modulus and Poisson's ratio of the plate material (aluminum) and $\nu_{s}$ the Poisson's ratio of the steel ball.

Strain induced in the plate can then be roughly estimated by $\varepsilon \sim p_{0} / E$. The ball displacement can be written as $d=d_{0}+\Delta d$, with $d_{0}$ the static (equilibrium) displacement when the pump vibration is off and $\Delta d$ the displacement amplitude when the pump is activated. Considering the low frequency excitation of the pump ( $1 \mathrm{~Hz}$ here), quasistatic 
behavior is assumed at each instant and therefore from Eqs. (A1) and (A2) the strain can be expressed as $\varepsilon=\varepsilon_{0}+\Delta \varepsilon$, with

$$
\varepsilon_{0} \sim K d_{0}^{1 / 3}
$$

the static contact strain, with

$$
K=\frac{h}{\pi L E}\left(\frac{3 b E^{* 2} E_{s}}{2 R^{2}}\right)^{1 / 3}
$$

and

$$
\Delta \varepsilon \sim \frac{K d_{0}^{-2 / 3}}{3} \Delta d
$$

the amplitude of the strain variation when the pump vibration is active.

For experimental quantification, we have captured the ball motion using a high-resolution video camera (Canon EOS 80D). Obtained displacement values for the first defect position [conditions of Figs. 3(a) and 4(a)] were $d_{0}=44$ $\mathrm{mm}$ and $\Delta d=0.4 \mathrm{~mm}$. From Eq. (A5) and the data $h=1 \mathrm{~mm}, b=2.7 \mathrm{~cm}, L=30 \mathrm{~cm}$, and $R=5 \mathrm{~mm}$, this yields $\Delta \varepsilon \simeq 1.75 \times 10^{-5}$. Finally, from repeated recordings with decreasing pump amplitudes, the detection threshold (value of strain amplitude below which the defect does not show on the images) has been empirically estimated around $10^{-5}$.

\section{APPENDIX B: ESTIMATE OF THE STRAIN INDUCED BY THE PROBE WAVE}

Under the Kirchhoff-Love plate assumptions, radial and angular strains in axisymmetrical problems can be approximated as ${ }^{21}$

$$
\left\{\begin{array}{c}
\varepsilon_{r r}=-z \frac{\partial^{2} w}{\partial r^{2}} \\
\varepsilon_{\theta \theta}=-z \frac{1}{r} \frac{\partial w}{\partial r}
\end{array}\right.
$$

where $(r, \theta)$ are the polar coordinates and $w$ is the normal displacement of the median plane of the plate.

In the case of a flexural wave radiated from the origin of the coordinate system, $w$ can be expressed as $w(r, \omega)$ $=(A(\omega) / \sqrt{r}) e^{j(\omega t-k r)}$, with $k^{2}=\omega \sqrt{12 \rho\left(1-\nu^{2}\right) / E e^{2}}$, and $E, \nu, \rho, e$ the plate's Young's modulus, Poisson's ratio, density, and thickness, respectively, and $A(\omega)$ an undetermined amplitude term depending on frequency and source characteristics. Equation (B1) then yields after far-field assumption

$$
\left\{\begin{array}{l}
\varepsilon_{r r} \sim z k^{2} w \\
\varepsilon_{\theta \theta} \sim 0 .
\end{array}\right.
$$

The maximum strain $\varepsilon=\varepsilon_{r r}(e / 2)$ is obtained at the plate surface $(z=e / 2)$. Its experimental quantification will be obtained from point measurement of normal velocity $v=d w / d t=j \omega w$ using a laser vibrometer (Polytec OFV552). From Eq. (B2) we thus obtain (in amplitude)

$$
\varepsilon \sim \sqrt{\frac{3 \rho\left(1-\nu^{2}\right)}{E}} v .
$$

In the experimental conditions of Sec. IV, the normal velocity field amplitude of the first arriving wavepacket measured at the defect position is around $0.04 \mathrm{~mm} / \mathrm{s}$. Equation $(\mathrm{B} 3)$ then yields $\varepsilon \simeq 1.3 \times 10^{-8}$.

${ }^{1}$ S. R. Anton, D. J. Inman, and G. Park, "Reference-free damage detection using instantaneous baseline measurements," AIAA J. 47(8), 1952-1964 (2009).

${ }^{2}$ H. W. Park, H. Sohn, K. H. Law, and C. R. Farrar, "Time reversal active sensing for health monitoring of a composite plate," J. Sound Vib. 302(12), 50-66 (2007).

${ }^{3}$ H. Sohn, H. W. Park, K. H. Law, and C. R. Farrar, "Combination of a time reversal process and a consecutive outlier analysis for baseline-free damage diagnosis," J. Intell. Mater. Syst. Struct. 18(4), 335-346 (2007).

${ }^{4}$ B. Park, H. Sohn, C.-M. Yeum, and T. C. Truong, "Laser ultrasonic imaging and damage detection for a rotating structure," Struct. Health Mon. 12(5-6), 494-506 (2013).

${ }^{5}$ H. W. Park, S. B. Kim, and H. Sohn, "Understanding a time reversal process in Lamb wave propagation," Wave Motion 46(7), 451-467 (2009).

${ }^{6}$ L. Huang, L. Zeng, and J. Lin, "Baseline-free damage detection in composite plates based on the reciprocity principle," Smart Mater. Struct. 27(1), 015026 (2018).

${ }^{7}$ S. B. Kim and H. Sohn, "Instantaneous reference-free crack detection based on polarization characteristics of piezoelectric materials," Smart Mater. Struct. 16(6), 2375-2387 (2007).

${ }^{8}$ Y. Zhang, V. Tournat, O. Abraham, O. Durand, S. Letourneur, A. L. Duff, and B. Lascoup, "Nonlinear mixing of ultrasonic coda waves with lower frequency-swept pump waves for a global detection of defects in multiple scattering media," J. Appl. Phys. 113, 064905 (2013).

${ }^{9}$ P. Shokouhi, J. Rivière, C. R. Lake, P.-Y. Le Bas, and T. Ulrich, "Dynamic acousto-elastic testing of concrete with a coda-wave probe: Comparison with standard linear and nonlinear ultrasonic techniques," Ultrasonics 81, 59- 65 (2017).

${ }^{10}$ L. Chehami, E. Moulin, J. de Rosny, C. Prada, O. Bou Matar, F. Benmeddour, and J. Assaad, "Detection and localization of a defect in a reverberant plate using acoustic field correlation," J. Appl. Phys. 115(10), 104901 (2014).

${ }^{11}$ L. Chehami, E. Moulin, J. de Rosny, C. Prada, J. Assaad, and F. Benmeddour, "Experimental study of passive defect detection and localization in thin plates from noise correlation," Phys. Proc. 70, 322-325 (2015).

${ }^{12}$ L. Chehami, E. Moulin, J. de Rosny, C. Prada, E. Chatelet, G. Lacerra, K. Gryllias, and F. Massi, "Nonlinear secondary noise sources for passive defect detection using ultrasound sensors," J. Sound Vib. 386, 283-294 (2017).

${ }^{13}$ J. Rivière, G. Renaud, R. A. Guyer, and P. A. Johnson, "Pump and probe waves in dynamic acousto-elasticity: Comprehensive description and comparison with nonlinear elastic theories," J. Appl. Phys. 114(5), 054905 (2013).

${ }^{14}$ Y. Zhang, O. Abraham, V. Tournat, A. L. Duff, B. Lascoup, A. Loukili, F. Grondin, and O. Durand, "Validation of a thermal bias control technique for coda wave interferometry (CWI)," Ultrasonics 53, 658-664 (2013).

${ }^{15}$ M. Lott, C. Payan, V. Garnier, P. Y. Le Bas, T. J. Ulrich, and M. C. Remillieux, "Three-dimensional modeling and numerical predictions of multimodal nonlinear behavior in damaged concrete blocks," J. Acoust. Soc. Am. 144(3), 1154-1159 (2018).

${ }^{16}$ J. N. Eiras, C. Payan, S. Rakotonarivo, and V. Garnier, "Damage detection and localization from linear and nonlinear global vibration features in concrete slabs subjected to localized thermal damage," Struct. Health Monit. 20(0), 567-579 (2021). 


\section{JASA}

${ }^{17}$ H. Achdjian, E. Moulin, F. Benmeddour, J. Assaad, L. Dupont, and L. Chehami, "Reverberation of flexural waves scattered by a local heterogeneity in a plate," J. Acoust. Soc. Am. 140, 157-164 (2016).

${ }^{18}$ J. Rivière, G. Renaud, S. Haupert, M. Talmant, P. Laugier, and P. A. Johnson, "Nonlinear acoustic resonances to probe a threaded interface," J. Appl. Phys. 107(12), 124901 (2010).
${ }^{19} \mathrm{~S}$. Timoshenko, Strength of Materials, 2nd ed. (Van Nostrand, New York, 1940), Vol. 1.

${ }^{20} \mathrm{~K}$. L. Johnson, Contact Mechanics (Cambridge University Press, Cambridge, UK, 1985).

${ }^{21}$ A. W. Leissa, Vibration of Plates (NASA, Washington, DC, 1969). 